\title{
Cold atmospheric plasma risk assessment: stem cells
}

\begin{abstract}
Regarding the fact that cell shape indicates cell health and is of particular importance in the evaluation of new therapies, in this study, stem cell deformation during Atmospheric Pressure Plasma (APP) treatment was investigated. Given that, cell deformation is a warning of cell damage, it is therefore expected that APP-based therapy, a new modern technology that is expanding worldwide, will not lead to the deformation of normal cells. Here, the stem cells exposed to Helium-fed jet plasma, with two di erent powers of 15 and $25 \mathrm{~W}$. Moreover, the duration of exposure was changed (30, 50, 70, and 90 seconds) to determine the most appropriate exposure time and voltage, which maintains stem cells' health condition. First of all, it was found that cold plasma at low power does not change the shape and elongation of stem cells. Besides, it was found that if the power of a cold plasma source is $25 \mathrm{~W}$, it will raise cell growth rate. In this paper, the gas ow rate of the helium plasma jet was set to 3.9 liters per minute, and a plasma source frequency of $30 \mathrm{kHz}$ was selected.
\end{abstract}

Keywords: cold atmospheric plasma, risk assessment, stem cell, fractal factor
Volume 7 Issue 4 - 2020

R. Jamshidi, K. Hajizadeh

Department of Physics, Islamic Azad University, South Tehran Branch, Iran

Correspondence: Kobra Hajizadeh, Department of Physics, South Tehran Branch, Islamic Azad University, Tehran, Iran, Tel +9891243955I0,Email k_hajizadeh@azad.ac.ir

Received: October 20, 2020 | Published: November 24, 2020

\section{Introduction}

Plasma electro-surgical systems have long been used for coagulation, tissue cutting, drying, and more. ${ }^{1}$ Despite all their conveniences, the problem with these technologies is tissue warming, meaning that their effects are more thermal. ${ }^{2}$ Recently, signi cant advances have been made in the science and engineering of atmospheric pressure plasma (CAP), which controls the temperature of the CAP and operates at temperatures below $40^{\circ} \mathrm{C}$. Over the past decade, a new innovative eld has emerged from the combination of plasma physics, life sciences, and clinical medicine known as plasma medicine. ${ }^{3,4}$ Today, thanks to modern atmospheric discharge systems and CAP-based medicine, ${ }^{5}$ utilizing plasma systems in-vivo has become possible, that is, plasma-based therapies have emerged. The purpose of this field is to employ the effects of cold plasma by managing the interaction between plasma components with specific structural elements of living cells. ${ }^{6,7}$ Recent advances in comprehending physical plasma phenomena, along with the development of new plasma sources, have paved the way for cold plasma therapies, with great potential in skin rejuvenation, disinfection, tissue regeneration, and wound healing. ${ }^{8}$ Plasma active components such as molecules, atoms, ions, electrons and photons, reactive species, UV, and heat enables controlling and catalyzing complex reactions and biochemical methods. ${ }^{9}$ Thermal and non-thermal plasma is now widely used in medicine. ${ }^{10}$

Recently, plasma medicine has become an innovative research eld with high potential for healing, a development that has resulted in a better understanding of plasma phe-nomena, and, consequently, the development of new plasma sources over the past few years. ${ }^{11}$ While thermal plasma is still used in various medical elds (for example, for burning and sterilizing medical devices), current research focuses mainly on the use of non-thermal plasma. Experiments show that Cold Atmospheric Plasma (CAP) enables e ective, non-contact, painless treatments, even at the microscopic level, without damaging healthy tissue. ${ }^{12}$ CAP can affect biochemical processes. In skin therapies, a new horizon has been opened to wound healing, tissue regeneration, skin infection treatment, and possibly other di cult-to-treat diseases. The first clinical trial demonstrated the superiority, efficiency, and tolerability of cold plasma in the treatment of chronically infected wounds. ${ }^{13,14} \mathrm{An}$ essential task is the introduction of plasma into clinical medicine provided that the so-called plasma-based therapy does not a ect the health of normal cells. Regarding the potentially targeted cells in wound healing, that is, the normal stem cells, their health after plasma exposure, is of vital importance.

Accordingly, to study cell health conditions, we managed to investigate the effects of helium plasma on normal stem cells at separate powers and exposure times in this paper. Cell viability was first evaluated by cell metabolic assay, and then cell deformation following helium plasma treatment was assessed by determining the cell fractal number. The purpose of this research was to ascertain the health range of plasma treated cells, which was well determined that in low-power regions of the generator, plasma treatment does not lead to cell deformation and damage. Moreover, helium plasma was found to enhance cell growth rate.

\section{Materials and methods}

\section{Plasma device}

The CAP device employed in this study was a He-Jet device, a central powered electrode inside the quartz tube. The voltage of plasma generator was set to 2.5 and $3 \mathrm{kV}$, in turn, and the exposure time was changed from $30-80 \mathrm{~s}$. The frequency of the discharge was $30 \mathrm{kHz}$, for the inlet industrial grade Helium with a ow rate of $3.91 / \mathrm{min}$. The power of the plasma-generator was around $5 \mathrm{~W}$. This device produces atmospheric plasma at a few degrees above room temperature thereby generating a mix of active components such as charged particles (electrons, ions), reactive oxygen and nitrogen species (ROS) and UV photons. The plasma jet was applied directly to the cells with a distance of $25 \mathrm{~mm}$ between the nozzle and the culture media. The CAP device used in this experiment is schematically shown in Figure 1.

\section{Optical emission spectroscopy}

The optical emission spectrum showed emission in the UVA, UVB, and UVC range. Nevertheless, most of the UV emission appeared in the UVB range, so that only minimal UVC emission was detected. The optical emission spectrum of the plasma discharge moreover showed that NO is present in the plasma system as the lines in the UVC region correspond to NO molecules (Figure 2). 


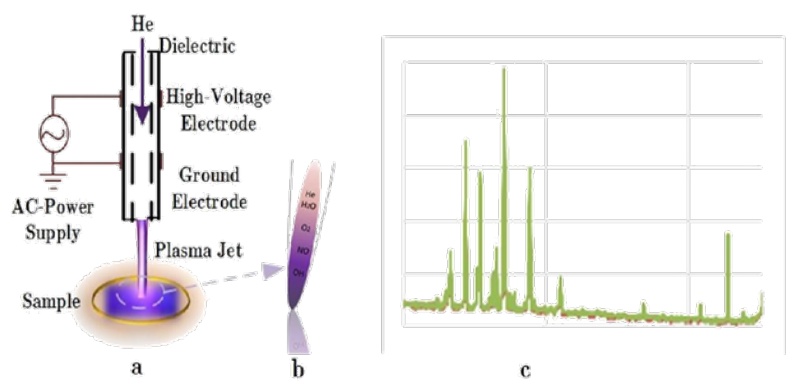

Figure I a) Schematic of the Plasma Jet have been used in this experiment b) Tip of the jet c) plasma spectra.

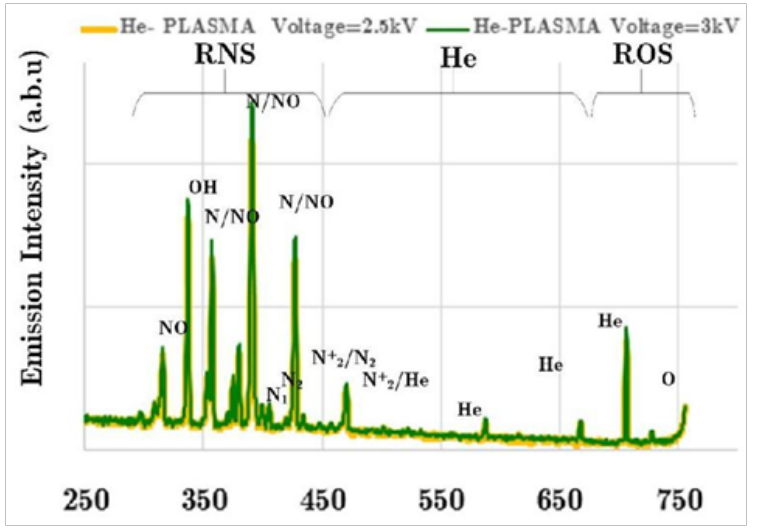

Figure 2 Optical Emission Spectrum of Helium Jet Plasma.

\section{Cell culture conditions}

Sample preparation: Stem cells, SCs, were provided by Bon-Yachte Co. To be treated with CAP, cells were seeded in $35 \mathrm{~mm}$ Petri dishes and were cultured in DMEM-F12 (Sigma-Aldrich) supplemented with 10\%FBS (fetal bovine serum, Sigma-Aldrich), 1œpenicillin, and strep-tomycin. The cells were maintained at $37^{\circ} \mathrm{C}$ in a humidified incubator, containing $5 \mathrm{eO}_{2}$. The medium was replaced every other day and ve days after seeding the cells were treated Helium-Jet Plasma $30 \mathrm{kHz}, 5 \mathrm{w}$, in which the gas ow rate was $3.91 \mathrm{~min}-1$ and the exposure time was 30-90s. The conuence of all Petri dishes was confirmed at about $80-90 \%$. All Petri dishes were further incubated once more at $37^{\circ} \mathrm{C}$ for 24 hours in the presence of 500 lit DMEM-F12.

\section{Rons assessment in plasma-activated-water (PAW)}

After treatment, the concentrations of free radicals such as $\mathrm{H}_{2} \mathrm{O}_{2}$ and $\mathrm{NO}_{2} / \mathrm{NO}_{3}$ were measured in plasma activated media by methyl orange (Sigma Aldrich) and nitrite/nitrate colorimetric assay kits, respectively. Some of the free radicals, for instance, $\mathrm{H}_{2} \mathrm{O}_{2}$ do not exist in after-glow productions (Figure 2, the photo spectroscopy emission). Conse-quently, the presence of these free radicals in Plasma Activated Medium, PAM is owed to the interaction of radicals and atoms with air molecules or water vapor molecules. Using a UV-visible spectrometer facilitated measuring the amount of these radicals quantitively, as can be seen in Figure 3.

\section{MTT assay}

Using the MTT [-[4,5-dimethylthiazol-2-yl]-2,5dyphenyltetrazolium bromide] assay, a colorimetric method to measure the activity of mitochondria and cellular dehydrogenase enzymes, cell viability was monitored. MTT is reduced to its insoluble formazan by dehydrogenase enzyme, giving a purple color, as can be seen in Figure 4.

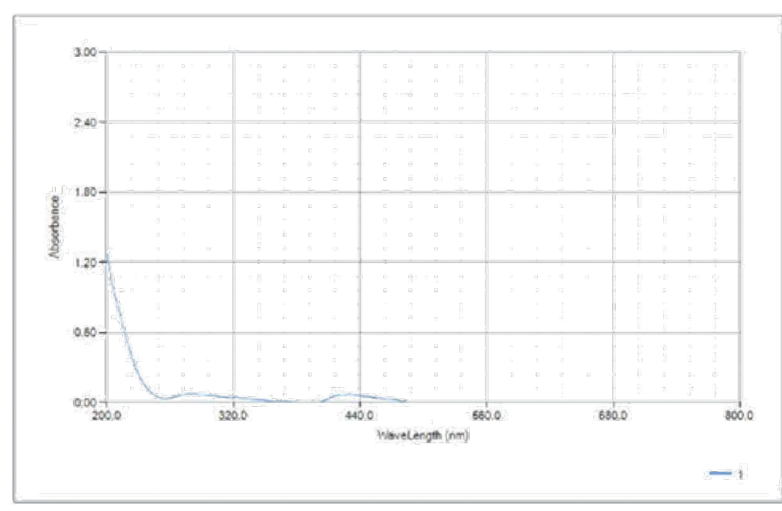

Figure 3 The result of UV-visible spectrometry of plasma activated media.

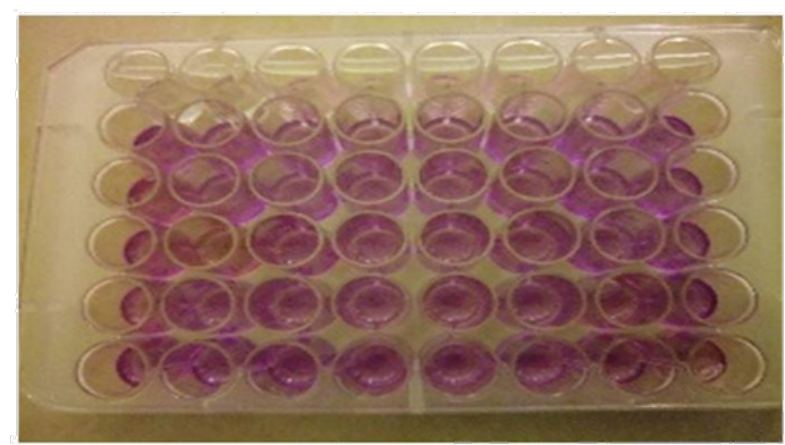

Figure 4 MTT-Assay results, the OE of each well shows the metabolic activity of cells.

\section{Fractal number}

The objective nature of fractal analysis and the strong correlation between tumor shape and its malignancy with the corresponding fractal dimension have made fractal analysis a suitable tool for morphological evaluation of cells in healthy growth and neoplasm one. Changes in cell shape lead to a change in mesenchymal cell differentiation. On the other hand, exposing force changes the morphology and consequently changes the behavior of the cell. ${ }^{15,16}$ In fact, the histological architecture changes while the intercellular adhesion increases. Besides, phase transitions from solid-state to liquid take place. Mechanical energy (cell adhesion of tumor tissue), which is mainly related to intracellular connections, has two components:

1- Contraction energy is attributed to the cytoskeleton and minimizes the intracellular area (Euclidean, when predominant, quasisolid), 2-Adhesion energy, which maximizes the surface (fractal, when predominant, quasi-liquid). Figure 5 shows the deformation of the cell and the change in the intercellular surface under mechanical pressure. This mechanical stress can be caused by cellular signaling or other mechanical stresses, including the collision of plasma components with them. As can be seen, the cell under high pressure deforms, and its shape changes. Technically, the cell structure switches from Euclidean to the fractal, as shown schematically in Figure 6.

Therefore, a variation in the fractal number (which represents the cell topology) reveals changes in the cell morphology and also cell behavior. The fractal number is a dimen-sionless quantity that indicates the dramatic effect of cell shape and environment on cell health, that is, a measurable quantity provided to better understanding about how cell damage and deformation (increase in cell entropy) spread. This quantity can predict the onset of cancer. 

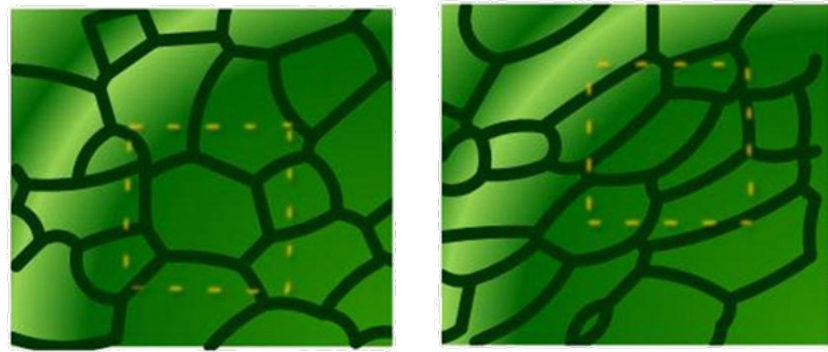

Figure 5 Deformation of the cell structure under pressure, a) normal cell b) deformed cell structure. ${ }^{17}$

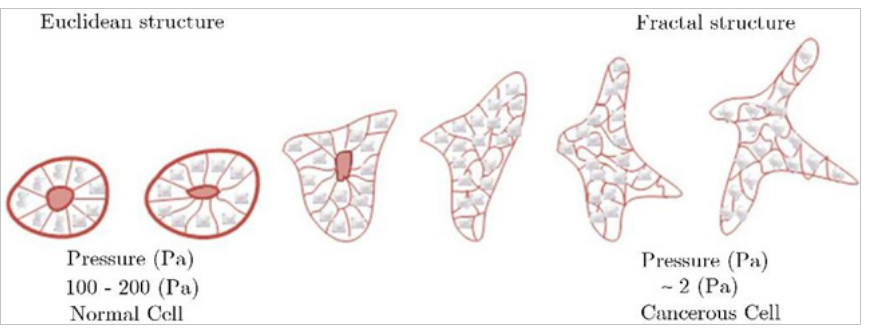

Figure 6 The continuous and reversible shifts from one structure to another. $^{18}$

Fractal number, FD, which represents cell entropy, and image complexity can be calculated from various methods, the most common of which are Sand-Box and Box-Counting methods. In this study, the Box-Counting method was used.

\section{Statistical analysis}

Use has been made of a Microsoft Excel software to plot the results, as mean standard deviation, and studentt-test to check the statistical signi cance $(* \mathrm{p}<0.05, * * \mathrm{p}<0.01, * * * \mathrm{p}<0.001)$.

Cells were seeded in at-bottomed 96-well plates at 2001 per well in each well, then incubated for two days to ensure proper adhesion and stability of the cells. The conuence of each well assured to be at $90 \%$. The cells were washed with phosphate buffer salt (PBS, Lonza $17-512 \mathrm{~F})$ and replaced with the fresh medium before being treated with cold plasma.

\section{Fractal factors evaluation}

Shape deformation assay was used to evaluate the e ects of cold plasma on normal Stem Cells. To this end, the bottom of the $35 \mathrm{~mm}$ petri dishes was covered with gelatin where the stem cells were then cultured. After three days, the cells were washed by PBS (Phosphate Buffer Salt), and the cell culture media replaced. After changing cell culture media, the dishes were exposed to plasma, with two di erent powers and di erent exposure time, the process is schematically shown in Figure 4. After 2 days, use has been made of MTT assay to evaluate the metabolic activity of stem cells. Three petri dishes were kept as controls. After plasma treatment, the petri dishes were incubated for 4 hours, after which the cells were photographed every hour by a CCD camera attached to a Nikon Invert microscope. Imaging results show that cells in plasma-treated petri dishes, grow faster than control dishes. The first column of Figure 7, shows the controlled petri-dishes, for both 2.5 (a) and $3 \mathrm{kV}$ (b) plasma generator, the next columns are exposed to plasma for $30,50,70$, and 90 s, respectively.

The metabolic activity, or cell viability is shown in Figure 8, as can be seen when the voltage of plasma generator is $3 \mathrm{kV}$, the cells grow faster, dose dependently (here, the duration of plasma exposure is considered as plasma dose).

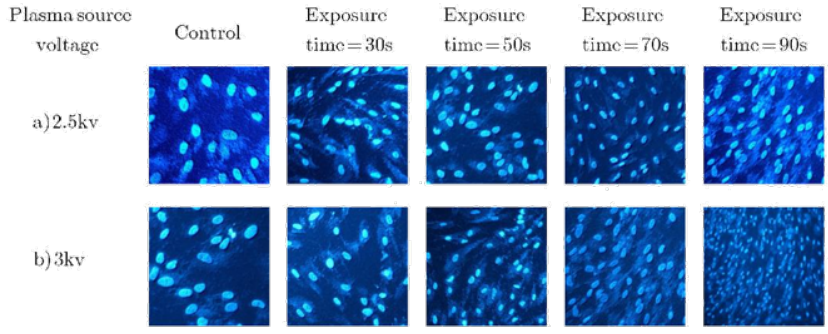

Figure 7 Stem Cells exposed to He-fed jet plasma, the plasma generator set to a) $2.5 \mathrm{kV}$ and b) $3 \mathrm{kV}$.

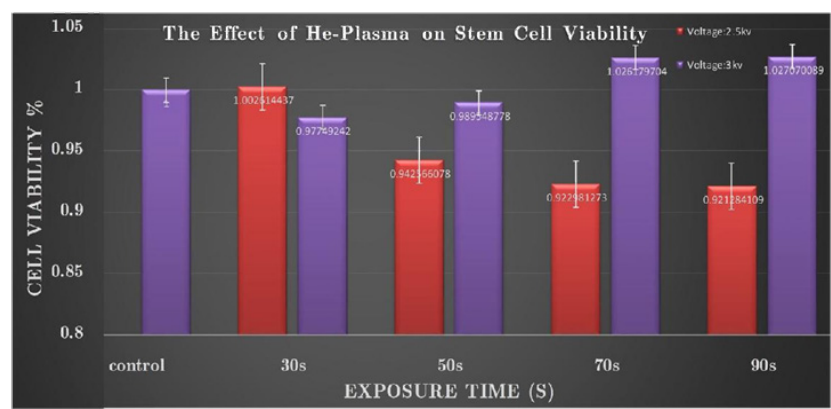

Figure 8 Cells viability before and after plasma treatment. As can be seen plasma caused a raise in cell growth, by increasing the exposure time the cell growth rate is increased $(* * p<0.01)$.

\section{Results and discussion}

According to extensive research on Fractal Factors, an increase in FD is associated with the onset of cancer. Recent theories about cancer stem cells suggest a link between the presence of self-regenerating subpopulations in tumor cells and tumorigenesis and indicate the ability of subpopulations to initiate metastatic proliferation. As a result, not changing the fractal factor is good news for plasma. It shows that the plasma has no effect on cell damage or, more importantly, the onset of cancer.

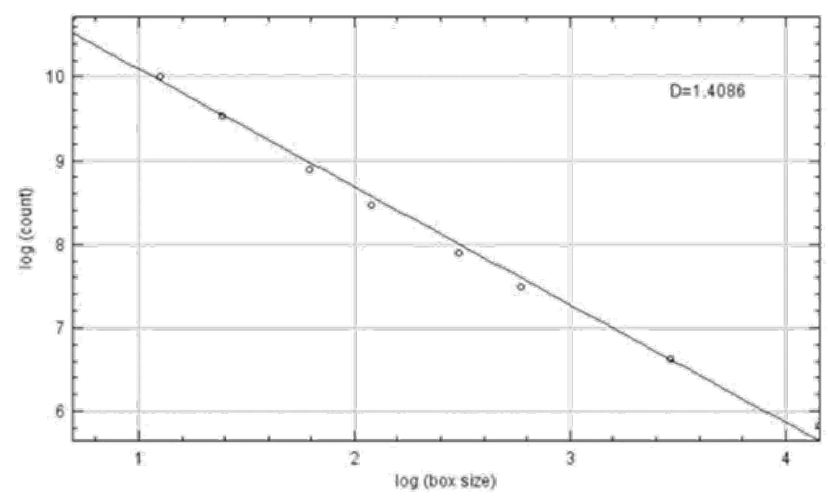

Figure 9 Fractal dimension of non-treated cell, FD=I.4086.

Plasma free radicals can convert PUFA (Poly Unsaturated Fatty Acid) to viscous liquids through chain polymerization, thus restricting the chain motion. Plasma exposure, and the production of free radicals in the cell culture medium, increases the viscosity of the medium. Perhaps at higher concentrations of RONS, free radicals can change the membrane uidity and increase its sti ness, which may be due to the interaction of free radicals with PUFAs..$^{19,20}$ According to the present study, it can be concluded that due to the amount of RONS produced in the cell culture medium, there was no signi cant change in cell 
sti ness and viscosity of the environment, and as a result, the fractal factor remained almost unchanged, which is a good sign, and shows the success of cold plasma-based treatments. However, this may not happen at higher powers. It is expected that by increasing RONS, the stiffness of the cell-substrate increases ${ }^{21}$ and leads to cell damage.
Fractal Dimension Fractal dimension (FD) evaluation shows no meaningful change in FD number. As can be seen in Table 1, the fractal number of control cell, Figure 9, and treated cells did not change significantly, therefore it can be deduced that cells did not deform after exposing to plasma with different power and different exposure times.

Table I The Fractal Dimension of treated stem cells did not show significant changes in comparison with control cells $\left({ }^{*} p<0.05\right)$

\begin{tabular}{llllll}
\hline Plasma source & Control & Exposure & Exposure & Exposure & Exposure \\
\hline voltage & & time $=30 \mathrm{~s}$ & time $=50 \mathrm{~s}$ & time $=70 \mathrm{~s}$ & time $=90 \mathrm{~s}$ \\
$2.5 \mathrm{kv}$ & 1.4086 & 1.4488 & 1.4688 & 1.4697 & 1.4917 \\
$3 \mathrm{kv}$ & 1.4086 & 1.4774 & 1.4683 & $1.477 \mathrm{I}$ & 1.4957 \\
\hline
\end{tabular}

\section{Conclusions}

Generally, an essential precondition for full acceptance of a new treatment method, in addition to academic, economic, and social studies as well as evidence-based studies, is a scientific analysis based on its risks and side effects. Given the extensive applications of cold atmospheric plasma, CAP, in medicine, the public debate about the possibility of plasma-associated risk is of vital importance. In the present study, it can be shown that the local application of CAP is classified as safe due to no changes in the Fractal number. In terms of biological safety, future studies could cover the long-term evaluation of CAP based therapies. Besides, differences in local conditions and employed systems must be considered. Particularly for clinicians who have not studied plasma physics, the sum of initial in vitro and in vivo research should eventually lead to indication of the specific dose.

In summary, the results with the helium plasma jet system showed no changes in the fractal factor number during plasma treatment. As a result, no significant risk potential is expected. The effects of ultraviolet light and temperature during cell plasma interactions can be ignored. Moreover, cell growth increased, which can be essential in plasma based therapies.

\section{References}

1. Auer JA, Stick JA. Equine Surgery. UK: Elsevier Health Sciences; 2018. p. 3104.

2. Martinsen T, Pettersen FJ, Kalv H, et al. Electrosurgery and temperature increase in tissue with a passive metal implant. Front Surg. 2019;6:8.

3. Heinlin J, Isbary G, Stolz W, et al. Plasma applications in medicine with a special focus on dermatology. J Eur Acad Dermatol Venereol. 2011 ;25(1):1-11.

4. Isbary G, Morfil G, Zimmermann J, et al. Cold atmospheric plasma: a successful treatment of lesions in hailey-hailey disease. Arch Dermatol. 2011;147(4):388-390.

5. Braithwaite NSJ. Introduction to gas discharges. Plasma Sources Science and Technology. 2000;9(4):517.

6. Suchentrunk R, Staudigl G, Jonke D, et al. Industrial applications for plasma processes examples and trends. Surface and Coatings Technology. 1997;1(3):1-9.

7. Weltmann K, Von Woedtke T. Plasma medicine current state of research and medical application. Plasma Physics and Controlled Fusion. 2016;59(1): 014031.
8. Von Woedtke T, Metelmann HR, Weltmann KD. Clinical plasma medicine: state and perspectives of in vivo application of cold atmospheric plasma. Contributions to Plasma Physics. 2014;54(2):104.

9. Xu RG, Chen Z, Keidar M, et al. The impact of radicals in cold atmospheric plasma on the structural modification of gap junction: A reactive molecular dynamics study. International Journal of Smart and Nano Materials. 2018.

10. Li Y, Kang MH, Uhm HS, et al. Effects of atmospheric-pressure nonthermal bio-compatible plasma and plasma activated nitric oxide water on cervical cancer cells. Sci Rep. 2017;(7):45781.

11. Graves DB. Reactive species from cold atmospheric plasma: implications for cancer therapy. Plasma Processes and Polymers. 2014;11(12):1120 1127.

12. Von Woedtke T, Reuter S, Masur K, et al. Plasmas for Medicine. Physics Reports. 2013;530(4):291-320.

13. Gentile RD. Cool atmospheric plasma (j-plasma) and new options for facial con-touring and skin rejuvenation of the heavy face and neck. Facial Plast Surg. 2018;34(1):66-74.

14. Isbary G, Zimmermann J, Shimizu T, et al. Non-thermal plasmamore than ve years of clinical experience. Clinical Plasma Medicine. 2013;1(1):19-23.

15. Jean RP, Gray DS, Spector AA. Characterization of the nuclear deformation caused by changes in endothelial cell shape. J Biomech Eng. 2004;126(5): 552-558.

16. Clause KC, Liu LJ, Tobita K. Directed stem cell differentiation: the role of physical forces. Cell Commun Adhes. 2010 Apr;17(2):48-54

17. Hammer D, Reinhart-King C, Paszek M, et al. Traction force microscopy reveals basic mechano-chemical principles of cell adhesion, speading and cell-cell organization. American chemical society. 2005;229(1):648.

18. Weaver VM. Adhesion-linked protein tyrosine phosphatases, morphogenesis and breast cancer progression. tech. rep., pennsylvania univ philadelphia, 2006.

19. Cheeseman K, Slater T. An introduction to free radical biochemistry. $\mathrm{Br}$ Med Bull. 1993;49(3):481-493.

20. Petersen RC. Free-radicals and advanced chemistries involved in cell membrane organization influence oxygen diffusion and pathology treatment. AIMS Biophy. 2017;4(2):240-283.

21. Chuang YC, Chang HM, Li CY, et al. Reactive Oxygen Species and Inflammatory Responses of Macrophages to Substrates with Physiological Stiffness. ACS Applied Materials \& Interfaces. 2020. 